\title{
MANOMETER TUBES FOR MONITORING COASTAL WATER LEVELS: NEW FREQUENCY RESPONSE FACTORS
}

\author{
A. Jafari ${ }^{\dagger 1}$, N. Cartwright ${ }^{\dagger}$, P. Nielsen ${ }^{\dagger}$ \\ $\dagger$ Griffith School of Engineering, Gold Coast Campus, Griffith University, Southport QLD 4215 Australia, a.jafari@griffith.edu.au, \\ n.cartwright@griffith.edu.au \\ $\$$ School of Civil Engineering, The University of Queensland, Brisbane St Lucia, QLD 4072, Australia, p.nielsen@uq.edu.au
}

\begin{abstract}
This paper presents findings of new laboratory experiments on the frequency response of semi-rigid manometer tubes for use in monitoring coastal hydrodynamic processes, extending and improving upon the previous work of Nielsen et al. (1993). An adjustable speed rotary electromotor was used to drive a known water level variation at one end of the tube and the pressure was measured at both ends of the tube, enabling a direct determination of the tube's frequency response. Testing was conducted over a range of frequencies $(0.0067 \mathrm{~Hz}<f<2 \mathrm{~Hz})$ and tube lengths $(10 \mathrm{~m}<L<900 \mathrm{~m})$. New frequency response factors were determined by fitting the semi-empirical gain function equation of Nielsen et al. (1993) to the observed gain data. New predictive formulas for the empirical coefficients are provided which yields substantial improvements over the existing constants deduced by Nielsen et al. (1993).
\end{abstract}

Keywords: Manometer tubes, coastal water level monitoring, Gain Function, frequency response function

\section{Introduction}

The ability to accurately monitor water levels in the coastal environment is essential across the fields of Engineering, Environmental and Geophysical Sciences. Whilst technological advances have addressed the issue of accuracy, the resultant products are costly and, in particular, their use under extreme weather conditions is risky. Nielsen(1988) first introduced the idea of using manometer tubes to monitor coastal water levels as both a cheap, but importantly, robust and accurate system for use in all conditions. Illustrated in Figure 1, this system consists of an array of semi-rigid nylon manometer tubes extended from land to the water and weighted down with chain. At the seaward end, the tubes are screened to prevent blockage by sand and at the landward ends the tubes terminate into either a glass riser for manual observation of the mean water surface or into a manifold with pressure transducers attached to capture high frequency pressure fluctuations induced by waves.

${ }^{1}$ Corresponding author. Tel.: +61 (07)55527608; fax: +61 (07) 555 28065. E-mail addresses:

a.jafari@griffith.edu.au(A. Jafari), Postal Address: Griffith School of Engineering, Gold Coast Campus, Griffith University, QLD 4222, Australia 


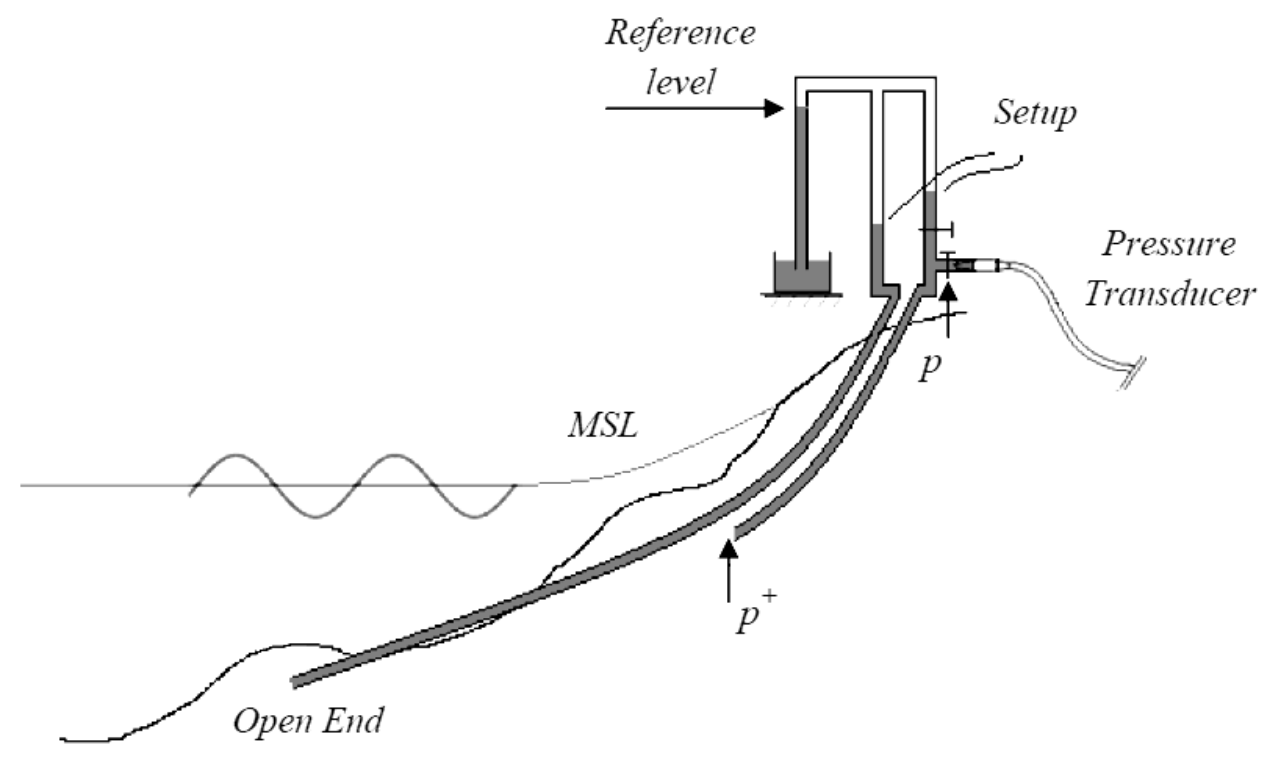

Figure 1. Schematic illustration of the manometer system of Nielsen(1988)

Numerous researchers have successfully used open-ended manometer tube systems to monitor the hydrodynamic characteristics of coastal and estuarine water bodies (e.g. Nielsen, 1988; Schubert and Bokuniewicz, 1991; Hanslow and Nielsen, 1993; Aagaard et al., 1994; Jafari et al., 2010; Jafari et al., 2011). However, the use of tubes closed by the connection of pressure transducers to monitor waves has had only limited application under field conditions (e.g. Nielsen et al., 1993; Hanslow et al., 1995; Jafari et al., 2011).

\section{Theoretical background}

This section outlines the theoretical development of Nielsen et al. (1993) (see also Nielsen and Dunn, 1998). A water wave-induced pressure wave inside the tube is reflected by the transducer at the landward end which sets up a "quarter length" resonating system. The frequency response function for such a system can be approximated by,

$$
F(f)=\frac{1}{\cos \left(\frac{\pi}{2} \frac{f}{f_{0}}\right)+i D_{E}\left(\frac{f}{f_{0}}\right)}
$$

where $f$ is the frequency of the pressure fluctuations, $f_{0}$ is the lowest resonant frequency of the tube i.e. $f_{0}=c / 4 L$ and $D_{E}\left(f / f_{0}\right)$ is an energy dissipation function. This can be used to extract both gain and phase information via $|F|$ and $\operatorname{Arg}(F)$ respectively. In this application however, only wave statics were of interest and so only the gain was examined. The gain function corresponding to equation (1) is,

$$
G(f)=|F(f)|=\frac{1}{\sqrt{\cos ^{2}\left(\frac{\pi}{2} \frac{f}{f_{0}}\right)+D_{E}^{2}\left(\frac{f}{f_{0}}\right)}}
$$


Energy dissipation in the tubes is caused by (a) energy dissipation inside the fluid and (b) energy absorbed by the deformation of the tube wall. The energy lost due to viscosity over one wave period in fluid can be estimated as follows (Nielsen et al., 1993),

$$
D E_{\text {Fluid }} \approx \frac{R_{O}^{2}}{E h} \sqrt{\omega v} L T
$$

where $R_{0}$ is the initial uniform tube radius, $E$ is the elastic modulus of the tube, $h$ is tube wall thickness, $\omega$ is the angular frequency, $v$ is the kinematic viscosity of the fluid, $L$ is the tube length and $T$ is the wave period.

The energy damping due to flexing of the tube wall is estimated as (Nielsen et al., 1993),

$$
D E_{\text {wall }} \approx \frac{|p|^{2} R_{O}^{3}}{E h} L \approx \frac{|p|^{2} R_{O}^{2}}{\rho c^{2}} L
$$

where, $c$ is wave celerity in tube, $\rho$ fluid density, and $p$ is excitation pressure.

Combining equations (3) and (4) plus given that input energy at the open end over one wave period corresponds to $p^{+} c R_{0}^{2} T$, where $p^{+}$is seaward wave pressure at open end, Nielsen et al.(1993) expressed the relative energy loss as,

$$
\mathrm{DE}=\frac{\text { energyloss }}{\text { energy input }}=G \frac{|p| L}{\rho c^{3}}\left[C_{1} f+C_{2} \frac{\sqrt{v f}}{R_{0}}\right]
$$

where, $C_{1}$ and $C_{2}$ are dimensionless coefficients and the gain, $G$ is the ratio of the pressures at each end of the tube i.e. $p=G \times p^{+}$(see Figure 1).

Inserting equation (5) into equation (2) yields the following implicit expression for the tube's gain function,

$$
G=\frac{1}{\sqrt{\left(\cos ^{2}\left(\frac{\pi \times f}{2 f_{o}}\right)+G^{2}\left[\frac{|p| L}{\rho c^{3}}\left(\left[C_{1} f+C_{2} \frac{\sqrt{v f}}{R_{0}}\right]\right)\right]^{2}\right)}}
$$

Based on the general form of equation(6), Nielsen et al.(1993) proposed the following semiempirical, explicit gain function,

$$
G=\frac{1}{\sqrt{\left(\cos ^{2}\left(\frac{\pi \times f}{2 f_{o}}\right)+\left[B_{1} \frac{L \times f}{c}\left(1+B_{2} \sqrt{\frac{v}{f \times I D^{2}}}\right)\right]^{2}\right)}}
$$


where $I D$ is the internal tube diameter, $v$ is kinematic viscosity (for water $v=1.0 \times 10^{-6} \mathrm{~m}^{2} / \mathrm{s}$ ) and the empirical coefficients $B_{1}$ and $B_{2}$ are found via calibration of equation (7) with laboratory data and $c$ is speed of waves sound in flexible tubes given by,

$$
c=\frac{1}{\sqrt{\rho(S+D)}}
$$

where $S$ is the compressibility of the fluid $\left(4.4 \times 10^{-10} \mathrm{~Pa}^{-1}\right)$ and $D$ is the distensibility of the tube $\left(1.4 \times 10^{-8} \mathrm{~Pa}^{-1}\right)$.

Nielsen et al. (1993) undertook laboratory calibration on two semi-rigid nylon tubes of lengths $59 \mathrm{~m}$ and $120 \mathrm{~m}$ and found best fit values for $B_{1}$ and $B_{2}$ to be the constants 0.58 and 5.0 respectively. The agreement between equation (7) and their calibration data is shown in Figure 2. It is noted that although these values provide good agreement for both tube lengths tested, it is expected that this will not be the case for longer tube lengths and so the present study extends the work of Nielsen et al. (1993) to cover a much wider range of tube lengths $(10 \mathrm{~m}<L<900 \mathrm{~m})$.

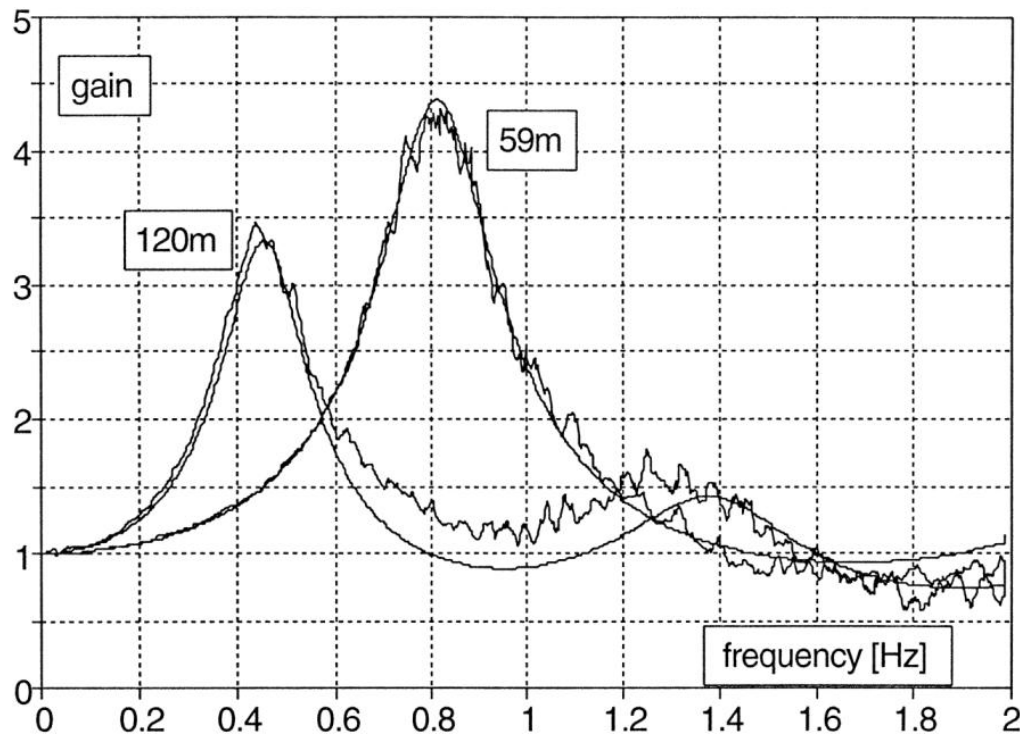

Figure 2. Gain function for 120m and 59m tube length (from Nielsen et al., 1993). The smooth curve is equation (7) and the noisy curve shows the data

\section{Approach}

\subsection{Experimental setup}

The basic concept for the empirical calibration is to induce a dynamic pressure fluctuation at the open ("seaward" end) ends of the tubes and then to measure the pressures at both ends of the tubes. The ratio of the two signals is the response of the tube system at that particular frequency and so repeated tests over a wide range of oscillation frequencies $(0.0050 \mathrm{~Hz}<\mathrm{f}<$ $2.0 \mathrm{~Hz}$ in this case) yields data on the system's frequency response function as exemplified by Figure 2. 
The presence of air in the system can drastically alter the response of the tube system due to the air's compressibility (Nielsen and Dunn, 1998) and so a small amount of dye was added to the water to facilitate a clearer view of the water in the translucent tubing to ensure no air was present. A gravity head of about $1.8 \mathrm{~m}$ was used to fill the shorter tubes $(L<100 \mathrm{~m})$ and for the longer tubes a suction pump was used to provide additional pressure head. Table 1 summarises the properties of the tubes that have been used.

Figure 3, illustrates the experimental setup. The dynamic pressure signal at the "seaward" end was induced by oscillating a water reservoir with a simple-harmonic rotating arm driven by a variable speed electromotor. The "seaward" end pressure was measured using a secondary tube so that measurement of any reflection back from the "landward" transducer (PT2) was avoided. The range of oscillation provided by the arm was $80 \mathrm{~cm}$. Vibration noise from the electro-motor was filtered from the signal and unwanted noise from the movement of the tubes hanging from the reservoir was minimised using a plastic box filled with water placed at the point that tubes touched the ground. It was found during the higher frequency testing $(f$ $>0.3 \mathrm{~Hz}$ ), the vibration noise from the electro-motor increased significantly due to the high speed of rotation and could not be adequately filtered and so for these frequencies manual excitation of the reservoir was adopted.

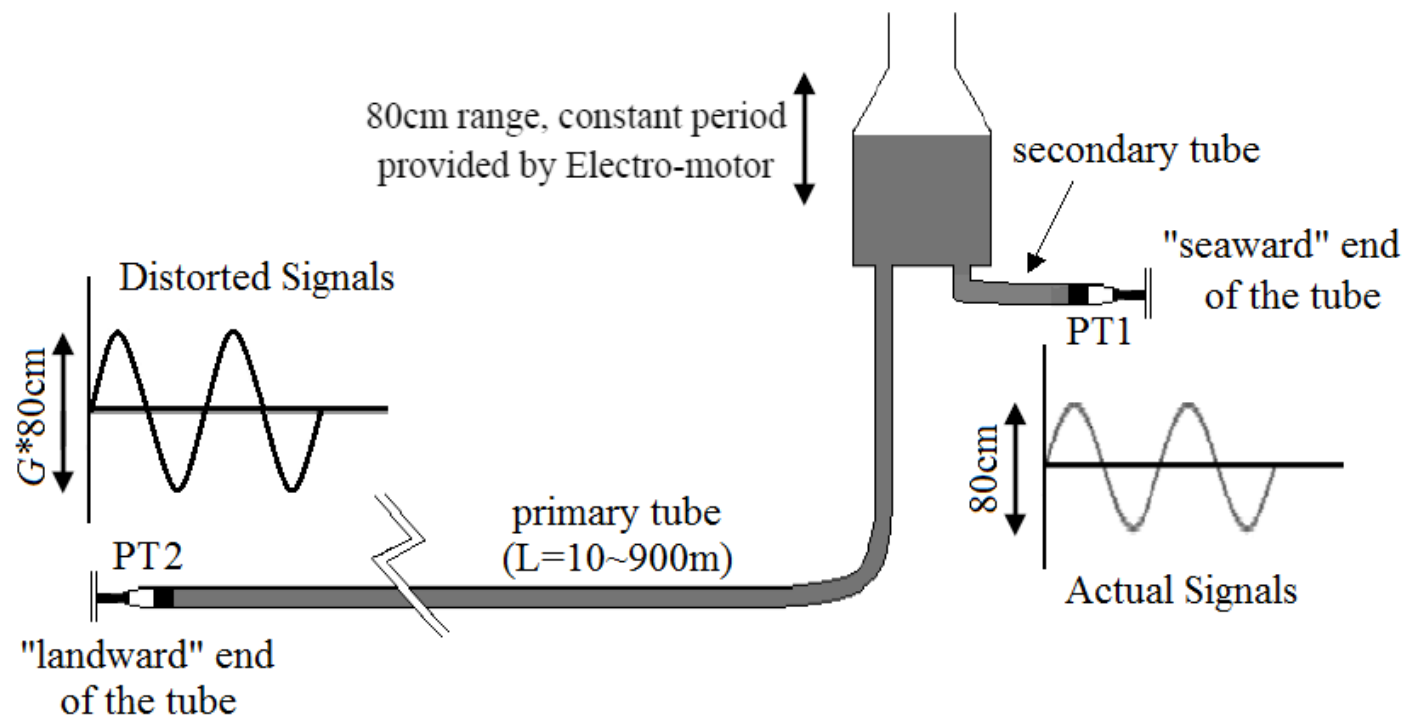

Figure 3. Sketch of the experimental setup where PT1 is the pressure transducer for recording the actual input signal representing the "seaward" end of the tube and PT2 is the sensor recording the resultant "landward" end signal $(G \times 80 \mathrm{~cm})$

\subsection{Data Analysis}

The raw times series from the two ends of the tubes were initially analysed using both time domain (zero-crossing) and frequency domain (spectral) methods to assess whether the results were sensitive to analysis method. The system gain $(G)$ was then computed as the ratio of $|p| /\left|p^{+}\right|$, where $|p|$ and $\left|p^{+}\right|$are the magnitudes of the pressure fluctuations at the "landward" (PT2) and "seaward" (PT1) ends of the tube respectively (see Figure 1 and Figure 3). An 
example comparison for two tube lengths is shown in Figure 4 and it is seen that there is little difference between both methods with the maximum difference being $2.0 \%$.

Table 1. Tube properties that used to extract gain function, where $L=$ tube length, $O D=$ outside diameter, $I D=$ inside diameter, $t=$ tube wall thickness, $c=$ speed of sound, and $f_{0}=$ lowest resonant frequency

\begin{tabular}{cccccc}
\hline \hline $\boldsymbol{L}(\mathbf{m})$ & $\boldsymbol{O D}(\mathbf{m})$ & $\boldsymbol{I D}(\mathbf{m})$ & $\boldsymbol{t}(\mathbf{m})$ & $\boldsymbol{c}(\mathbf{m} / \mathbf{s})$ & $\boldsymbol{f}_{\boldsymbol{0}}(\mathbf{H z})$ \\
\hline 10 & 0.008 & 0.0054 & 0.0012 & 403.0 & 10.075 \\
20 & 0.008 & 0.0054 & 0.0012 & 403.0 & 5.037 \\
30 & 0.008 & 0.0054 & 0.0012 & 403.0 & 3.358 \\
40 & 0.008 & 0.0054 & 0.0012 & 403.0 & 2.519 \\
50 & 0.008 & 0.0054 & 0.0012 & 403.0 & 2.015 \\
100 & 0.008 & 0.0054 & 0.0012 & 403.0 & 1.007 \\
350 & 0.01 & 0.007 & 0.0015 & 403.0 & 0.288 \\
750 & 0.01 & 0.007 & 0.0015 & 403.0 & 0.134 \\
900 & 0.01 & 0.007 & 0.0015 & 403.0 & 0.112 \\
\hline
\end{tabular}

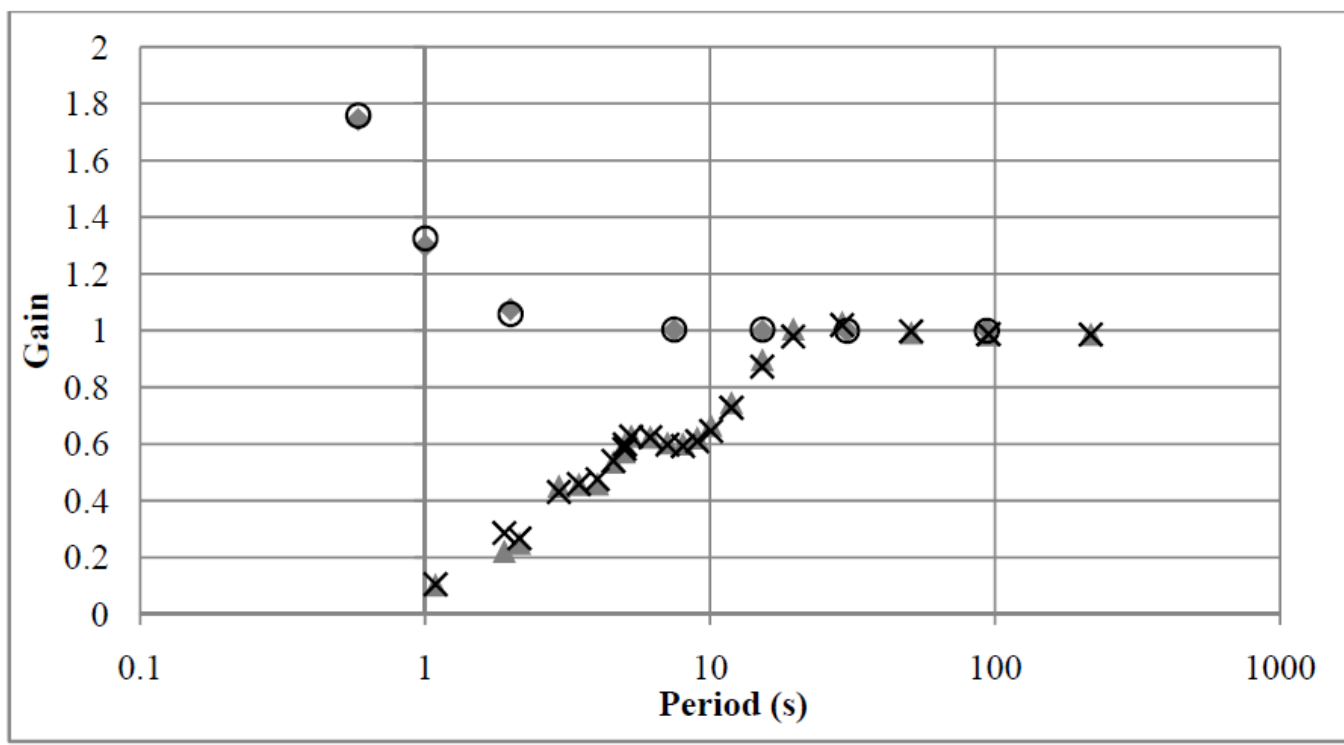

Figure 4. Comparison of time domain and spectral analysis results for $L=100 \mathrm{~m}$ (time domain ( $\bullet$ ), frequency domain $(\circ))$ and $L=900 \mathrm{~m}$ (time domain $(\boldsymbol{\Delta})$, frequency domain $(\times)$ ) tubes.

\section{Results and Discussion}

Figure 5 shows the initial comparison between the experimental data and equation(7) using the Nielsen et al. (1993) derived constants $B_{1}=0.58$ and $B_{2}=5.0$. For $L=750 \mathrm{~m}$ and $100 \mathrm{~m}$ (left and right respectively) and qualitative discrepancies are obvious. In order to improve the ability of equation (7) to reproduce the data a 2-step regression analysis was undertaken.

The first step entails an adjustment of the quarter-length resonant frequency, $f_{0}$, to account for the fact that the sensor diaphragms are not rigid but flex under pressure. In this situation a time lag, $\delta_{t}$, occurs in the resonance frequency which can be accounted for using (Nielsen et al., 1993), 


$$
f_{\text {resonance }}=\frac{1}{4 L / c+2 \delta_{t}}=\frac{f_{o}}{\left(1+c \delta_{t} / 2 L\right)}=\frac{f_{0}}{\Delta}
$$

where, $\Delta$ is adjusted such that the peak frequency according to equation (1) matches the gain's peak frequency in the data.

According to equation (9), $\Delta$ is a function of inverse tube length and speed of sound and therefore as the tube length increases makes the relative fraction, i.e. equation (9), smaller and ultimately $\Delta$ approaches to 1 and so $\Delta$ is more important for shorter tube lengths than longer ones. This is further evidenced by the best fit $\Delta$ values summarized in Table 2 .
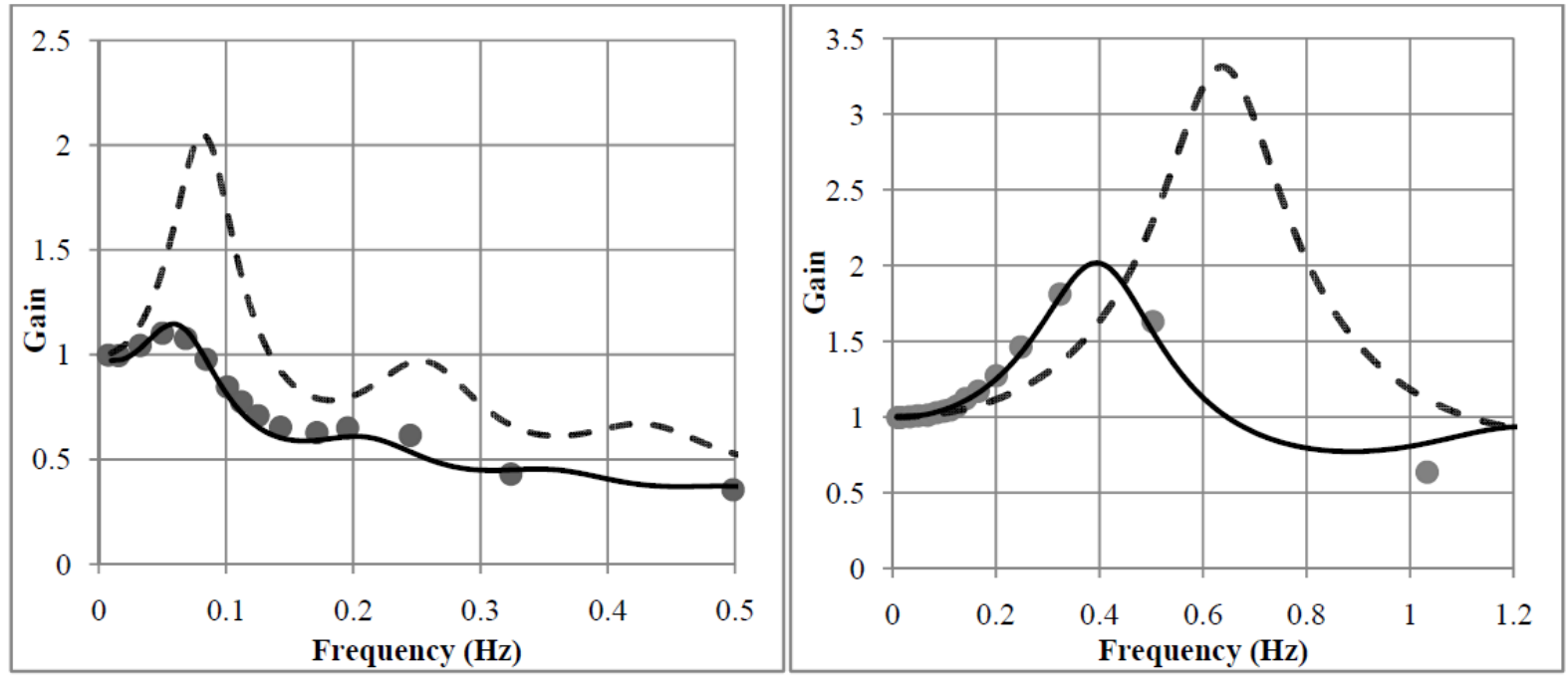

Figure 5. Comparison of equation (7) with experimental data $(\bullet)$ using Nielsen et al. (1993) constants $B_{1}=0.58$ and $B_{2}=5.0$ (dashed line) and the present best-fit variable $B_{1}, B_{2}$, and $\Delta$ values with modified $f_{0}$ (solid line) for $750 \mathrm{~m}$ (left) and $100 \mathrm{~m}$ (right) tubes.

Table 2. From left to right: tube length $(L)$, inside diameter $(I D)$, tube wall thickness $(t)$, peak frequency modifier $(\triangle)$, gain function empirical factors $\left(B_{1} \& B_{2}\right)$, regression coefficient of determination of applying presented $B_{1}, B_{2}$, and $\Delta$ in relation with lab data

\begin{tabular}{ccccccc}
\hline \hline $\boldsymbol{L}(\mathbf{m})$ & $\boldsymbol{I D}(\mathbf{m})$ & $\boldsymbol{t}(\mathbf{m})$ & $\Delta$ & $\boldsymbol{B}_{\boldsymbol{1}}$ & $\boldsymbol{B}_{\boldsymbol{2}}$ & $\mathbf{R}^{\mathbf{2}}$ \\
\hline 10 & 0.0054 & 0.0012 & 3.50 & 5.50 & 5.10 & 1.00 \\
20 & 0.0054 & 0.0012 & 2.40 & 2.75 & 5.80 & 0.91 \\
30 & 0.0054 & 0.0012 & 2.10 & 1.68 & 6.00 & 1.00 \\
40 & 0.0054 & 0.0012 & 2.00 & 1.25 & 6.30 & 0.92 \\
50 & 0.0054 & 0.0012 & 1.95 & 1.20 & 7.00 & 0.92 \\
100 & 0.0054 & 0.0012 & 1.55 & 1.00 & 7.80 & 0.98 \\
350 & 0.007 & 0.0015 & 1.25 & 0.50 & 12.90 & 0.99 \\
750 & 0.007 & 0.0015 & 1.20 & 0.31 & 25.00 & 0.98 \\
900 & 0.007 & 0.0015 & 1.20 & 0.26 & 31.00 & 0.95 \\
\hline
\end{tabular}

The second step is then to fit equation (7) with the modified $f_{0}$ to the data by least-squares regression adjustment of $B_{1}$ and $B_{2}$. The results from two of the tube lengths tested are shown 
in Figure 5. The best-fit values of $B_{1}$ and $B_{2}$ for all of the tubes tested are provided in Table 2 and it is evident that $B_{1}$ and $B_{2}$ are no longer constant for all tube lengths.

Curve fitting to the data in Table 2 was undertaken to establish predictive functions for $\Delta, B_{1}$, and $B_{2}$. Since the energy dissipation in the tube is primarily influenced by the tube dimensions (i.e. tube length and internal diameter), an appropriate choice of dimensionless independent variable is the ratio of tube length to internal diameter $(L / I D)$. The following equations are the best fit expressions to the data in Table 2 as plotted in Figure 6 and Figure 7.

$$
\begin{aligned}
& \Delta=16.789 \times\left(\frac{L}{I D}\right)^{-0.233} \\
& B_{1}=552.89\left(\frac{L}{I D}\right)^{-0.653} \\
& B_{2}=2 \times 10^{-4} \times\left(\frac{L}{I D}\right)+4.643
\end{aligned}
$$

Figure 6 and Figure 7 show the agreement between equations (10), (11) and (12) with $\Delta, B_{1}$ and $B_{2}$ values respectively which are presented in Table 2 . As shown in the figures, regression coefficient of determination shows high agreement between the predictive equations and these constant values.

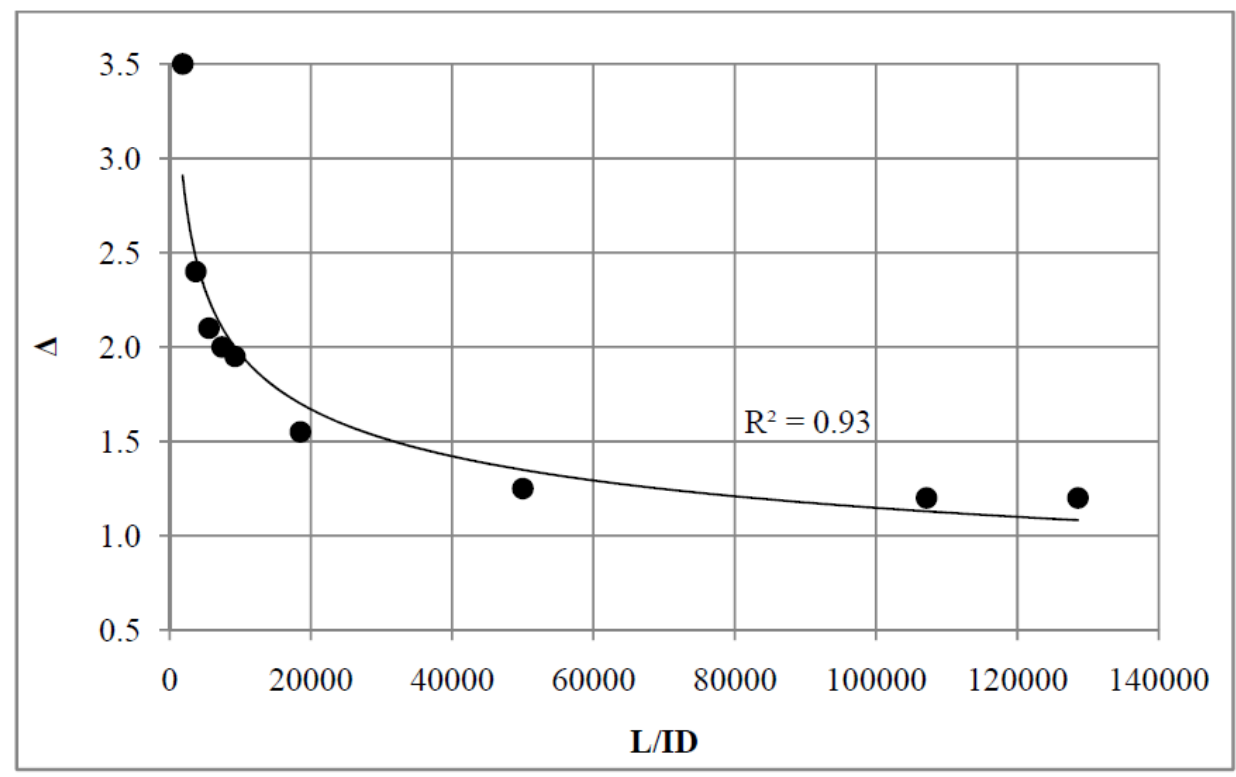

Figure 6. Agreement between values of $\Delta(\bullet)$ with equation(10) (solid line) 


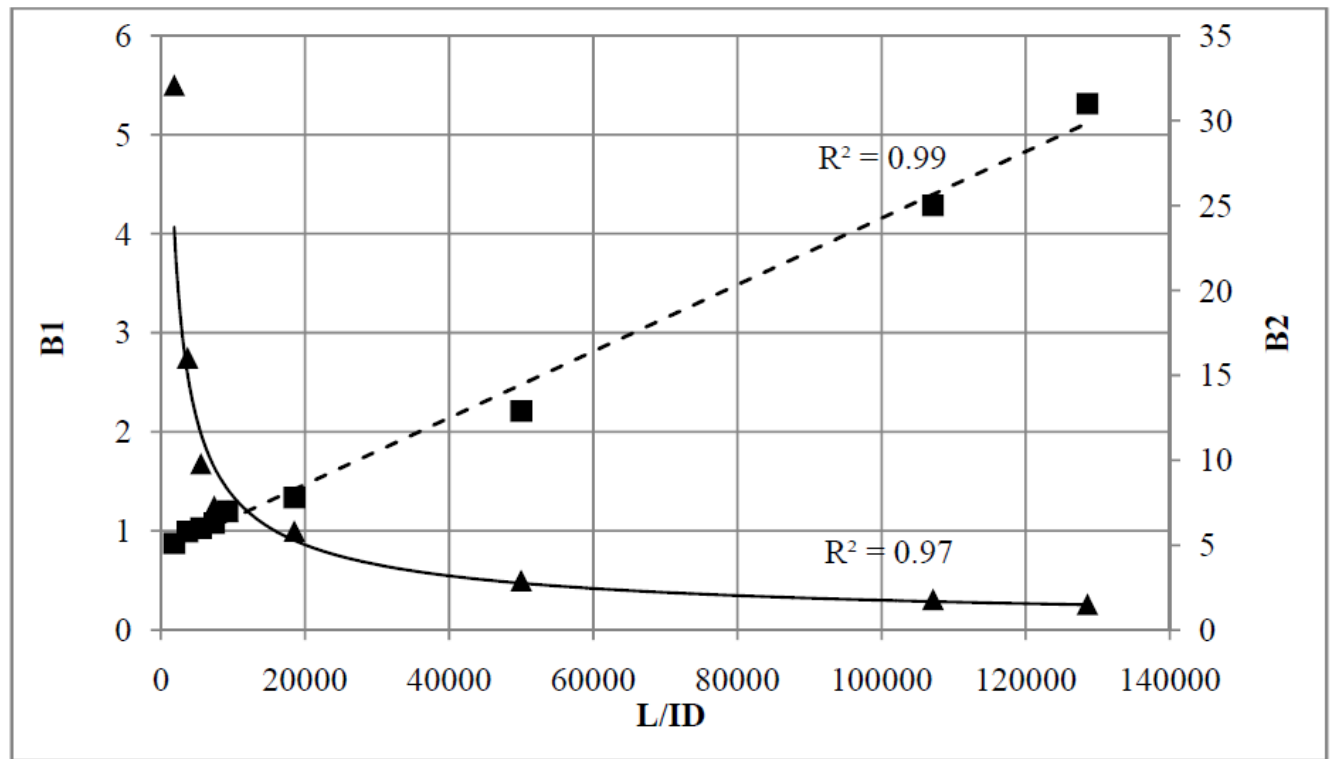

Figure 7. Agreement between values of $B_{l}(\boldsymbol{\Delta})$ with equation (11) (solid line) and $B_{2}(\boldsymbol{\square})$ with equation (12) (dashed line).

Inserting equations (10), (11) and (12) in to equation (7) yields,

$$
G=\frac{1}{\sqrt{\left(\cos ^{2}\left(\frac{\pi \times f}{2 f_{o}} \times 16.789 \times\left(\frac{L}{I D}\right)^{-0.233}\right)+\left[\left(552.89\left(\frac{L}{I D}\right)^{-0.653}\right) \frac{L \times f}{c}\left(1+\left(2 \times 10^{-4} \times\left(\frac{L}{I D}\right)+4.643\right) \sqrt{\frac{v}{f \times d^{2}}}\right)\right]^{2}\right.}}
$$

\section{Verification of Proposed Constant Equations}

Equation (13) was then verified by comparison with the laboratory data. Figure 8 provides an example comparison for the $750 \mathrm{~m}$ tube length which shows good agreement. Table 3 provides summary of coefficient of determination $\left(\mathrm{R}^{2}\right)$ for all tube lengths. For reference, the regression for equation (7) with Nielsen's constant $B_{1}$ and $B_{2}$ ( 0.58 and 5 respectively) is also shown. The significant improvement of equation (13) over the existing constant values of Nielsen et al. (1993) is clearly apparent.

The $40 \mathrm{~m}$ tube length has the poorest $\mathrm{R}^{2}$ value. This occurs mainly because of relatively low number of observations $(\mathrm{N}=9)$ and that one of the values (at $2.0 \mathrm{~Hz})$ there is an anomalous $42 \%$ difference between the predicted value and the recorded data. It is noted that the frequency of $2.0 \mathrm{~Hz}$ is outside of the range of frequencies normally expected in a natural sea state and if this anomalous data point is not considered in the regression the $\mathrm{R}^{2}$ is improved to a value of 1.00 (see Table 3). 


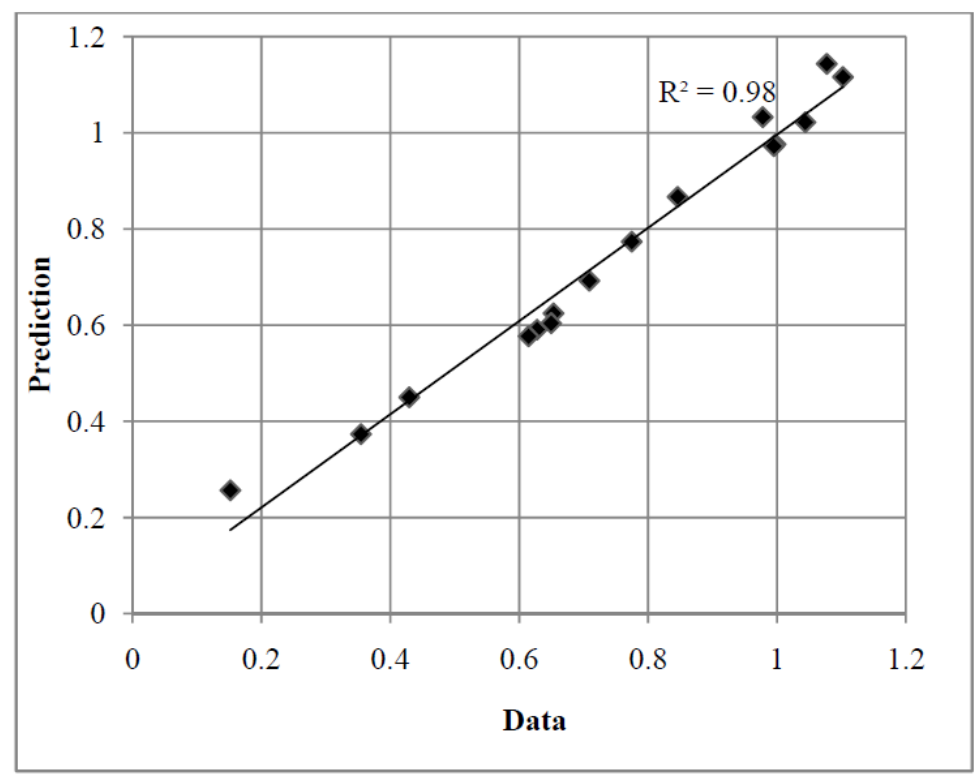

Figure 8 . Verifying Predictive values against recorded data for $750 \mathrm{~m}$ tube

Table 3. Summary of coefficient of determination $\left(\mathrm{R}^{2}\right)$, left to right: tube length $(L)$, Number of data points which shows the resolution of experiment $(N), R^{2}$ of equation(13), $R^{2}$ of equation(7), $\mathrm{R}^{2}$ of equation (13) in frequency band width of sea wave $(f<0.5 \mathrm{~Hz})$

\begin{tabular}{|c|c|c|c|c|}
\hline$L(\mathbf{m})$ & $\mathbf{N}$ & $\begin{array}{c}\mathbf{R}^{2} \\
\text { equation }(13) \\
(0.0067 \mathrm{~Hz}<f<2 \mathrm{~Hz})\end{array}$ & $\begin{array}{c}\mathbf{R}^{2} \\
\text { constant } B_{1} \& B_{2} \\
\text { (Nielsen et al., 1993) }\end{array}$ & $\begin{array}{c}\mathbf{R}^{2} \\
\text { equation(13) } \\
\text { (sea wave frequencies) }\end{array}$ \\
\hline 10 & 7 & 0.99 & 0.99 & 1.00 \\
\hline 20 & 8 & 0.90 & 0.10 & 1.00 \\
\hline 30 & 8 & 0.98 & 0.01 & 1.00 \\
\hline 40 & 9 & 0.62 & 0.02 & 1.00 \\
\hline 50 & 17 & 0.99 & 0.11 & 1.00 \\
\hline 100 & 16 & 0.81 & 0.41 & 1.00 \\
\hline 350 & 22 & 0.94 & 0.41 & 0.94 \\
\hline 750 & 17 & 0.98 & 0.60 & 0.98 \\
\hline 900 & 23 & 0.95 & 0.60 & 0.96 \\
\hline
\end{tabular}

\section{Conclusion}

A series of laboratory experiments were conducted to examine the frequency response of semi-rigid manometer tubes for use in monitoring coastal hydrodynamic processes. The data collected was used to establish new empirical factors for the semi-empirical gain function formula of Nielsen et al. (1993). The results provide marked improvement on the predictions made using constant derived of Nielsen et al. (1993) who used only two tube lengths.

The wide range of tube lengths tested in the current study facilitated the formulation of predictive equations for the empirical factors as a function of the tube geometry (length and diameter). The new predictive formulas in conjunction with Nielsen et al. (1993) gain 
function formula provide an accurate means to monitor coastal/surf zone water level variations under any climatic conditions.

\section{Acknowledgements}

The help and assistance of Mr. Ian Underhill and Mr. Duncan Ward through performing the laboratory test is appreciated. Funding for the present work and financial support for the first author has been provided by Australian Research Council grant number DP0877235.

\section{References}

Aagaard, T., Nielsen, N. and Nielsen, J., 1994. Cross-shore structure of infragravity standing wave motion and morphological adjustment: An example from northern zealand, denmark. Journal of coastal research, 10(3): 716-731.

Hanslow, D.J. and Nielsen, P., 1993. Wave setup on beaches and in river entrances. ASCE, NEW YORK, NY(USA). 1: 240-252.

Hanslow, D.J., Nielsen, P. and Hibbert, K., 1995. Measuring waves with manometer tubes. p.^pp. 207-218.

Jafari, A., Cartwright, N. and Nielsen, P., 2010. Extracting empirical factor of wave parameters based on filed data recorded under storm condition. International Conferences on Coasts, Ports, and Marine Structures, Tehran, Iran, p.^pp.

Jafari, A., Cartwright, N. and Nielsen, P., 2011. Stormy wave analysis based on recorded field data on south-east coasts of queensland, australia. Journal of coastal Research, SI 64: 527-533.

Nielsen, P., 1988. Wave setup: A field study. Journal of Geophysical Research, 93(No C12): 15643-15652.

Nielsen, P. and Dunn, S.L., 1998. Manometer tubes for coastal hydrodynamics investigations. Coastal Engineering, 35: 73-84.

Nielsen, P., Hanslow, D.J. and Apelt, C.J., 1993. A new type of nearshore wave gauge. 11th Aust. Conf. Coastal Ocean Eng. Townsville.: 247-251.

Schubert, C.E. and Bokuniewicz, H.J., 1991. Infragravity wave motion in a tidal inlet. Coastal sediments 91. ASCE, New York, p.^pp. pp. 1434-1446. 